\author{
MITSUBISHI ELECTRIC RESEARCH LABORATORIES \\ http://www.merl.com
}

\title{
Constant Modulus 4D Optimized Constellation Alternative for DP-8QAM
}

\author{
Kojima, K,; Millar, D.S.; Koike-Akino, T.; Parsons, K.
}

TR2014-083 September 2014

\begin{abstract}
We propose a constant modulus 4D format as an alternative to DP-8QAM. It has 0.4dB lower $\mathrm{SNR}$ at $\mathrm{BER}=10^{\wedge}-2$, and $1 \mathrm{~dB}$ higher span loss budget in nonlinear regime.

European Conference on Optical Communications (ECOC)
\end{abstract}

This work may not be copied or reproduced in whole or in part for any commercial purpose. Permission to copy in whole or in part without payment of fee is granted for nonprofit educational and research purposes provided that all such whole or partial copies include the following: a notice that such copying is by permission of Mitsubishi Electric Research Laboratories, Inc.; an acknowledgment of the authors and individual contributions to the work; and all applicable portions of the copyright notice. Copying, reproduction, or republishing for any other purpose shall require a license with payment of fee to Mitsubishi Electric Research Laboratories, Inc. All rights reserved.

201 Broadway, Cambridge, Massachusetts 02139 



\title{
Constant Modulus 4D Optimized Constellation Alternative for DP-8QAM
}

\author{
Keisuke Kojima ${ }^{(1)}$, David S. Millar ${ }^{(1)}$, Toshiaki Koike-Akino( ${ }^{(1)}$, Kieran Parsons $^{(1)}$ \\ (1) Mitsubishi Electric Research Labs., 201 Broadway, Cambridge, MA 02139, USA. kojima@merl.com
}

Abstract We propose a constant modulus 4D format as an alternative to DP-8QAM. It has 0.4dB lower $S N R$ at $B E R=10^{-2}$, and $1 \mathrm{~dB}$ higher span loss budget in nonlinear regime.

\section{Introduction}

Various modulation formats have been studied for coherent optical communications ${ }^{1}$. 8-aryquadrature amplitude modulation (8QAM) plays an important role by filling the gap between quaternary phase shift keying (QPSK) and 16QAM in terms of bit rates and reach ${ }^{2,3}$. It has also been proposed that 8 QAM-16QAM or QPSK$8 Q A M$ are used in time-domain hybrid ${ }^{4}$. In order to achieve similar bit rate with improved sensitivity, quaternary code or sphere-cut lattice eight-dimensional codes $^{5}$, and 4D honeycomb lattice $\operatorname{codes}^{6}$ have been proposed. However, for these 6bits/symbol codes, simultaneously achieving high sensitivity, Gray coding, and constant modulus has not been realized. In this work, we start with DP-8PSK which has the properties of constant modulus and Gray coding. Then on the Poincare sphere, we move the constellation points to increase Euclidean distance among the nearest neighbor words, while maintaining constant modulus. Transmission simulation results show that our proposed code, 4-dimensional 2-ary amplitude 8-ary phase shift keying (4D2A8PSK), has at least $1 \mathrm{~dB}$ better nonlinear performance than DP-8QAM.

\section{Constellation Design}

We start with the constellations projected onto the Stokes space. Figure 1(a) shows the dual polarization (DP)-8QAM constellation points in the Stokes space, where radius of each point represent the signal power. Since it is not constant modulus, the radius of each point differs significantly, and that affects the nonlinear performance. Figure $1(b)$ shows the dual polarization8-ary phase shift keying (DP-8PSK), where each projected points represents 8 words of 6 bits which are actually separated well in 4D space. The nearest words in the 4D space correspond to the nearest points in Fig. 1(b), so DP-8PSK has poor noise sensitivity. In order to increase the nearest points in the Stokes space, we came up with the constellation configuration in Fig. 1(c), where the 8 constellation points are staggered and separated into two groups. Our proposed 4D2A8PSK code can be expressed as

$$
\begin{gathered}
x(k, l)=a(l) e^{j \phi_{k}}, \quad y(k, l)=b(l) e^{j \phi_{l}} \\
\phi_{k}=\pi / 4 \times(k-1), \quad \phi_{l}=\pi / 4 \times(l-1) \\
k=1 . .8, \quad l=1 . .8
\end{gathered}
$$

where

$$
\begin{gathered}
a(l)=r_{1}, b(l)=r_{2}(k+l=\text { even }) \\
a(l)=r_{2}, b(l)=r_{1} \quad(k+l=\text { odd }) \\
r_{1}=\sin (\theta+\pi / 2) / \sqrt{2}, \quad r_{2}=\cos (\theta+\pi / 2) / \sqrt{2}
\end{gathered}
$$

The ratio of the two radii $r_{2} / r_{1}$ is equal to $\tan ^{-1}(\theta+\pi / 2)$. Note that $r_{2} / r_{1}=1$ for the DP8PSK case. The constellation for DP-8PSK and 4D-2A8PSK are shown in Fig. 2. As $\theta$ deviates from 0 , two rings in DP-8PSK and 4D-2A8PSK become separated. The main difference is that, if the amplitude in the x-polarization is larger, then that in the $y$-polarization is smaller, in order to keep constant modulus. The labeling of 4D2A8PSK follows that of DP-8PSK.

In order to understand the behavior of nearest neighbors, we plot a histogram of 4D Euclidean distances of all the constellation points as a function of $r_{2} / r_{1}$, as shown Fig. 3. Starting with $r_{2} / r_{1}=1$ (DP-8PSK), the count for the shortest distance combination remains the same until $r_{2} / r_{1}$ reaches 0.5858 , and this means that Gray coding is maintained for $r_{2} / r_{1}$ between 1 and close to 0.5858 .

Note that 4D-2A8PSK constellation in $\mathrm{x}$ - or $\mathrm{y}$ polarization alone looks similar to the 16 amplitude/phase shift keying (16APSK) used in wireless communications ${ }^{7}$, but 16APSK have not been used in the context of $4 \mathrm{D}$ codes. 
(a)

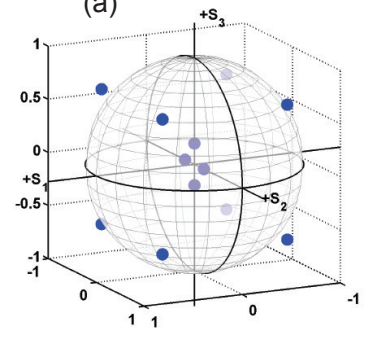

(b)

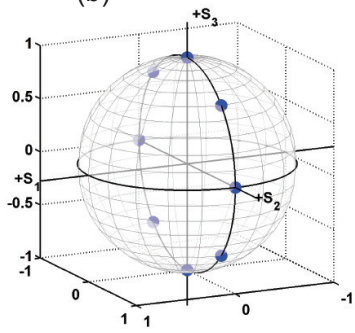

(c)

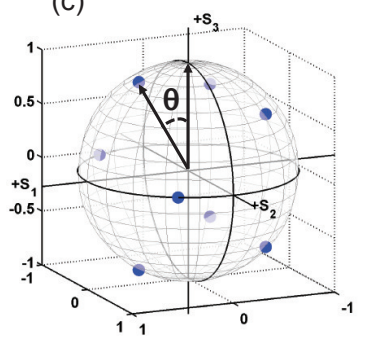

Fig. 1: Constellations in Stokes space: (a) DP-8QAM, (b) DP-8PSK, (c) 4D-2A8PSK

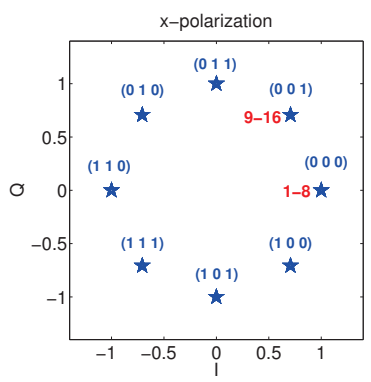

(a) 8PSK-X

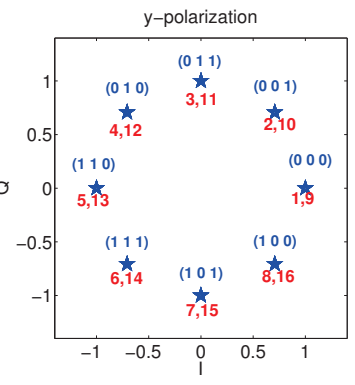

(b) 8PSK-y

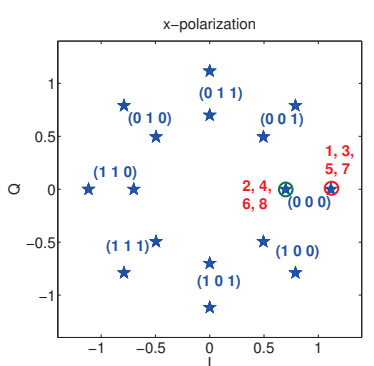

(c) 4D-2A8PSK-x

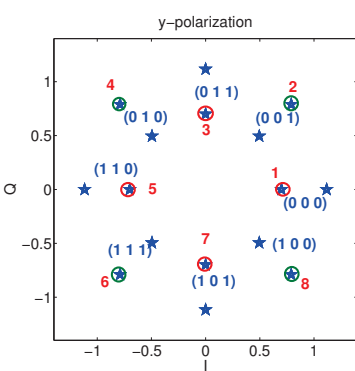

(d) 4D-2A8PSK-y

Fig. 2: Constellation of DP-8QAM and 4D-2A8PSK, where $\left(\begin{array}{lll}0 & 1 & 0\end{array}\right)$ etc. represent the half of the bits of the code word, and red numbers $1,2,3, \ldots$ represent the constellation points corresponding in $\mathrm{x}$ - and y-polarizations. Red and greed circles in (c) and (d) indicate corresponding points in $\mathrm{x}$ - and $\mathrm{y}$-polarizations

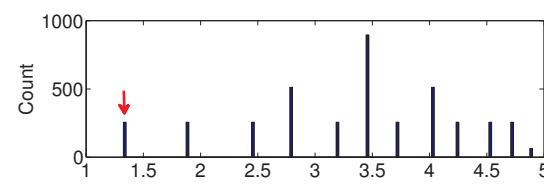

(a) $r_{1} / r_{2}=1$

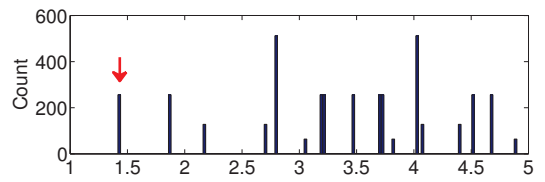

(b) $r_{1} / r_{2}=0.8$

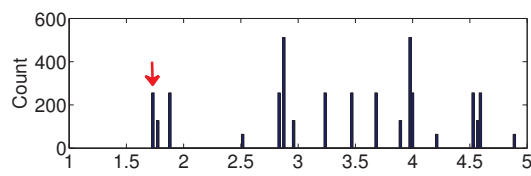

(c) $r_{1} / r_{2}=0.6$

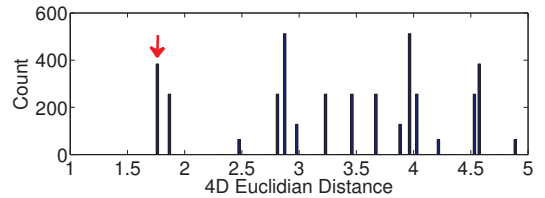

(d) $r_{1} / r_{2}=0.5858$

Fig. 3: Histogram of 4D Euclidean distaces of 4D-2A8PSK, for (a) $r_{1} / r_{2}=1$, (b) 0.8 , (c) 0.6 , and (d) 0.5858 . The red arrows show the smallest distances

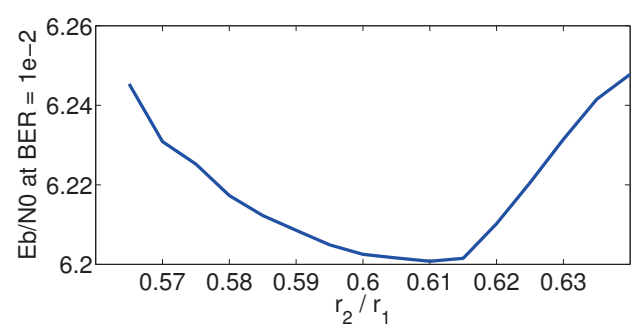

Fig. 4: $E_{b} / N_{0}$ vs. $r_{2} / r_{1}$ for the $4 \mathrm{D}-2 \mathrm{~A} 8 \mathrm{PSK}$ code

\section{Noise sensitivity}

We first evaluate additive white Gaussian noise (AWGN) channel performances of highdimensional modulation formats for signal-tonoise ratio (SNR) regime concerning uncoded BER thresholds of modern forward error correction (FEC) codes $^{9}$. SNR is defined as $E_{b} / N_{0}$ where $E_{b}$ is the energy per bit and $N_{0}$ is the unilateral power spectral density of the noise per dimension. BER vs. SNR curves obtained by Monte-Carlo simulations.

We numerically calculated $E_{b} / N_{0}$ which satisfies BER $=10^{-2}$, while changing $r_{2} / r_{1}$. As shown in Fig. $4, r_{2} / r_{1}=0.6-0.61$ gives the lowest BER under the nonlinear condition. We use $r_{2} / r_{1}=0.6$ for the remainder of this paper.

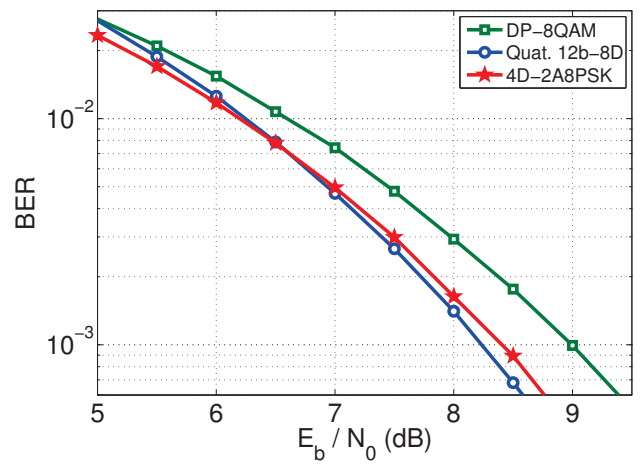

Fig. 5: BER vs $E_{b} / N_{0}$ for DP-8QAM, 4D-2A8PSK, and $12 \mathrm{~b}-8 \mathrm{D}$ codes.

Figure 5 shows the BER vs SNR for multiple codes. In addition to 4D-2A8PSK and DP-8QAM, 
we plotted quaternary 12 bit - 8 dimension code (Quat. 12b-8D) ${ }^{5}$, which is known to be the best high ( $>4$ ) dimensional code to our knowledge. This shows that 4D-2A8PSK has the best sensitivity among the 3 codes for a BER greater than $8 \times 10^{-3}$, while Quat. 12b-8D code offers superior performance at $\mathrm{BERs}$ below $8 \times 10^{-3}$. At $\mathrm{BER}$ $=1 \times 10^{-2}, 4 \mathrm{D}-2 \mathrm{~A} 8 \mathrm{PSK}$ and Quat. $12 \mathrm{~b}-8 \mathrm{D}$ codes have $0.39 \mathrm{~dB}$ and $0.57 \mathrm{~dB}$ gain, respectively, over DP-8QAM.

\section{Optical transmission performance}

We simulated transmission performance over a $6000 \mathrm{~km}$ standard single-mode fiber (SSMF) or non-zero dispersion shifted fiber (NZDSF) link at a rate of $125 \mathrm{~Gb} / \mathrm{s}$ per wavelength. Modulated symbols are mapped to the four dimensions (4D2A8PSK), eight dimensions (Quat. 12b-8D), and two-dimensions (DP-8QAM) ${ }^{5}$. At the transmitter, DP-I/Q modulators were driven by rectangular pulses, filtered by a $5^{\text {th }}$ order Bessel filter with $-3 \mathrm{~dB}$ bandwidth of 0.7 times the symbol rate. 5 wavelength channels with the same code were simulated with $50 \mathrm{GHz}$ spacing and no optical filtering. The link comprises 75 spans of $80 \mathrm{~km}$ SSMF or NZDSF with loss compensated by Erbium doped fiber amplifiers with 5.0 $\mathrm{dB}$ noise figure. In order to quantify performance over a single link for multiple modulation formats, span loss budget was used as a performance metric $^{8}$. SSMF parameters were as follows: $\gamma=1.2 \mathrm{~N} / \mathrm{km} ; D=17 \mathrm{ps} / \mathrm{nm} / \mathrm{km} ; \alpha=0.2 \mathrm{~dB} / \mathrm{km}$. NZDSF parameters were, $\gamma=1.6 / \mathrm{W} / \mathrm{km} ; D=3.9$ $\mathrm{ps} / \mathrm{nm} / \mathrm{km} ; \alpha=0.2 \mathrm{~dB} / \mathrm{km}$. Other fiber effects such as dispersion slope and polarization mode dispersion were not simulated. An ideal homodyne coherent receiver was used, with a transfer function described by a $5^{\text {th }}$ order Bessel filter with $-3 \mathrm{~dB}$ bandwidth 0.7 times the symbol rate, followed by sampling at twice the symbol rate. Following this, ideal chromatic dispersion equalization and dataaided least mean square equalization were employed. We assumed a BER threshold of $1 \times 10^{-2}$ for a $20 \%$ FEC $^{9}$. The plots for span loss budget vs. launch power for the three modulation formats are given in Fig. 6 for SSMF, and Fig. 7 for NZDSF. In the low launch power regime (-8 $\mathrm{dBm})$ where linear propagation effects are dominant, 4D-2A8PSK code gives $0.45 \mathrm{~dB}$ higher margin compared to DP-8QAM. The AWGN channel results predicted $0.4 \mathrm{~dB}$ improvement, and 0.05 $\mathrm{dB}$ discrepancy can be explained by the nonlinear effect in the case of SSMF. For higher launch pow- ers where nonlinearity is dominant, performance improvements become more significant, and the peak margin difference is above $1 \mathrm{~dB}$. The difference becomes more apparent in the case of NZDSF case. 4D-2A8PSK also performed better than the best $8 \mathrm{D}$ code with $3 \mathrm{bits} / \mathrm{s} / \mathrm{Hz} / \mathrm{pol}$ spectral efficiency.

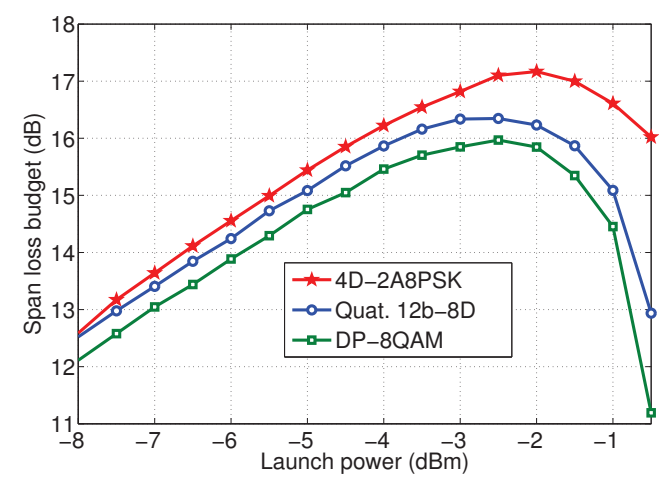

Fig. 6: Span loss budget vs launch power for 4D-2A8PSK, Quaternary $12 \mathrm{~b}-8 \mathrm{D}$, and DP-8QAM codes for 75 spans of $80 \mathrm{~km}$ SSMF at a target BER $=10^{-2}$.

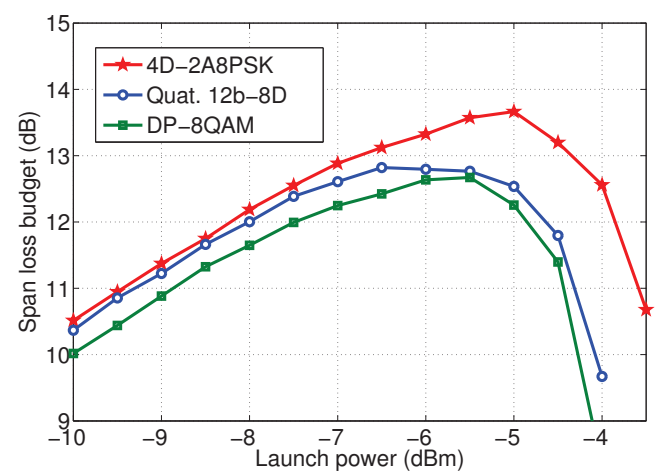

Fig. 7: Span loss budget vs launch power for 4D-2A8PSK, Quaternary 12b-8D, and DP-8QAM codes for 75spans of $80 \mathrm{~km}$ NZDSF at a target BER $=10^{-2}$.

\section{Conclusions}

We propose a constant modulus 4D-2A8PSK code. Compared to the DP-8QAM, this has 0.4 $\mathrm{dB}$ better sensitivity in linear region, while it has greater than $1 \mathrm{~dB}$ higher sensitivity in nonlinear region both for SSMF and NZDSF transmissions.

\section{References}

[1] E. Agrell and M. Karlsson, JLT 27, 5115 (2009).

[2] J. Zhang et al., Proc. OFC, Th4F.6 (2014).

[3] S. Zhang et al., Proc. OFC, W1A.3 (2014).

[4] Q. Zhuge et al., Proc. OFC, OTh4E (2013).

[5] D. S. Millar et al., Proc. OFC, M3A.4 (2014).

[6] H. Buelow et al.,Proc. OFC, M2A.6 (2014).

[7] F. Adachi and M. Sawahashi, IEEE Trans. Comm., 44, 416 (1996).

[8] Poggiolini et al., Opt. Exp. 8, 11360 (2010).

[9] F. Chang et al., IEEE Comm. Mag., 48, S48-S55, (2010). 